\title{
Maternal Risk Factors and Morbidity Pattern of Very Low Birth Weight Infants: A NICU Based Study at Eastern Nepal
}

\author{
Poudel P1, Budhathoki S², Shrivastava MK $^{3}$ \\ ${ }^{1}$ Dr. Prakash Poudel. MD, Assistant Professor, ${ }^{2}$ Dr. Sunil Budhathoki. Senior Resident, ${ }^{3}$ Dr. Manoj Kumar Shrivastava, \\ MD, Assistant Professor, All from the Department of Paediatrics, BP Koirala Institute of Health Sciences, Dharan, \\ Nepal.
}

Address of Correspondence: Dr. Prakash Paudel, E-mail: prakashpdl@hotmail.com

\begin{abstract}
Background: Preterm deliveries of babies weighing less than 1500 grams (VLBW, very low birth weight) are of major concern because of maximum perinatal morbidity and mortality found in this group. VLBW babies overload the neonatal intensive care unit (NICU) and their mortality contributes significantly to neonatal and infant mortality. Objectives: To study the common maternal risk factors associated with VLBW births. To study the morbidity and mortality patterns of VLBW babies. Methods: This was a hospital based retrospective study done in NICU of BP Koirala Institute of Health Sciences. Data were collected from medical records of VLBW babies admitted in NICU over a period of three years (13 April 2005 to 12 April 2008). Data were analyzed with SPSS 10.0 software. Results: Data of 140 VLBW babies were analyzed. Mean birth weight was $1188.9( \pm 212.78), 10.7 \%$ were home deliveries, $24.3 \%$ were twins, $31.4 \%$ required active resuscitation at birth and $46.4 \%$ were exposed to antenatal steroid. Common maternal risk factors associated with premature deliveries were inadequate antenatal check up (ANC) visits (95.7\%), twin pregnancy (24.3\%), antepartum hemorrhage (APH, 28.6\%), premature rupture of membrane $(23.6 \%)$, pregnancy induced hypertension $(12.9 \%)$, bad obstetric history (12.9\%) and maternal age less than 20 years $(8.6 \%)$. Common morbidities among VLBW babies were clinical sepsis $(77.1 \%)$, non physiological hyperbilirubinemia $(73.6 \%)$, apnea $(48.6 \%)$, shock (42.9\%), hypoglycemia(39.3\%), anemia (29.3\%), hyaline membrane disease (HMD, 21.4\%), patent ductus arteriosus $(15.0 \%)$, severe hypothermia $(12.1 \%)$, culture proven sepsis $(15.7 \%)$, retinopathy of prematurity (5.7\%), and bronchopulmonary dysplasia (3.6\%). Overall survival was $54.3 \%$ and major causes of death were HMD (51.0\%) and sepsis (34.7\%). Median durations of hospital stay were 17.5 days for survivors and five days for expired cases. Conclusion: Increasing the coverage of ANC visits, early diagnosis and treatment of APH and pregnancy induced hypertension, discouraging the childbirth at too young age, early diagnosis and treatment of acute infections and chronic medical diseases in mothers are important measures to decrease the burden of VLBW births. Common morbidities in VLBW babies are sepsis, HMD, apnea, hyperbilirubinemia, patent ductus arteriosus, shock, anemia, hypoglycemia, hypothermia and hypocalcemia. Common causes death of VLBW babies are HMD and sepsis. Overall survival of VLBW babies in our settings is less. More numbers of well equipped NICUs and services like surfactant therapy are needed to improve survival of VLBW infants in our set up.
\end{abstract}

Key words: Maternal risk factors, Morbidity, Mortality, Very low birth weight.

\section{Introduction}

P Koirala Institute of Health Sciences (BPKIHS) is a tertiary care centre and medical college located at eastern Nepal. It is running a seven bedded level II neonatal intensive care unit (NICU) with two neonatal ventilators. Advances in both perinatal and neonatal care over the past two decades mean that increasing numbers of preterm and very low birth weight (birth weight $<1500$ grams; VLBW) infants are surviving ${ }^{1}$. It is estimated that in Nepal, approximately two third of all deaths in first year of life occur in the first months of life. ${ }^{2}$ Of these deaths approximately two third occur in the first week of life. ${ }^{2}$ A significant number of these 
deaths may be contributed by deaths of VLBW infants. It is reported that $60.0 \%$ to $80.0 \%$ of neonatal mortality and morbidity is due to preterm birth ${ }^{3}$. In developing countries, neonatal mortality already accounts for more than $50.0 \%$ of the infant mortality coefficient ${ }^{4}$. So in order to improve infant mortality rate, it is essential to prevent preterm births and improve survival of neonates, especially preterm neonates. The consequence of preterm birth also leads to significant direct and indirect costs that have to be borne by parents and society. Hence a better understanding of antenatal factors contributing to preterm birth and morbidity pattern in very low birth weight infants are needed to improve the perinatal care. Local data on risks of preterm birth and morbidity pattern of VLBW infants will help to plan special care pattern for prevention and management of VLBW infants. Hence, we present this retrospective study of risks for VLBW birth and morbidity pattern in VLBW infants.

\section{Methods}

This was a retrospective and longitudinal study. The study was done in NICU of BPKIHS, Nepal. All VLBW infants were identified by reviewing the admission registry of NICU. Cases admitted between 13 April 2005 to 12 April 2008 were identified and their record files were obtained from medical record section. Inborn as well as outborn babies were included. Cases without documented birth weight (taken before 24 hours of life) and having grossly inadequate data were excluded. Medical records were reviewed and data extracted regarding perinatal characteristics, morbidity and mortality. Mothers with less than three antenatal check up (ANC) visits to health care facility were defined to have inadequate ANC visits. The gestational age (GA) was assessed using date of last menstrual period and confirmed by modified Ballard score method. In cases of gross discrepancy, Ballard score method was taken as valid. A birth weight less than $10^{\text {th }}$ percentile for GA was classified as small for gestational age $(\mathrm{SGA})^{5}$. Those babies who required resuscitation in the form of bag and mask ventilation or further steps were defined to have required active resuscitation at birth. Regarding Apgar score and use of maternal antenatal steroid, data were not available for few cases due to no documentation in home delivered and some out of hospital delivered babies. So those two parameters were analyzed separately including the cases with available data. Blood sugar level $<40 \mathrm{mg}$ per deciliter was defined as hypoglycemia. Ionized calcium $<4 \mathrm{mg}$ per deciliter was defined as hypocalcemia ${ }^{5}$. Axillary temperature below $35.5^{\circ} \mathrm{F}$ was defined as severe hypothermia as per WHO Integrated Management of Childhood Illness guideline. Any level of serum bilirubin requiring intervention was defined as non physiological hyperbilirubinemia ${ }^{5}$. Hematocrit level less than 30 was taken as anemia. Data were analyzed using SPSS 10.0 statistical software.

\section{Results}

There were 1135 admissions in NICU during three years. Among them 150 were VLBW infants. It was $13.2 \%$ of total NICU admissions. In 10 cases, there were grossly inadequate data. So, those 10 cases were excluded from the study. Final analysis was done in 140 cases. Mean birth weight was 1189.9 grams with standard deviation (SD) of 212.78.mean gestational age (GA) was 30.8 (SD 2.37) weeks. Among VLBW infants, 7.1\% were born before 28 weeks GA, 71.4\% were born between 28 weeks to 32 weeks GA, and $21.4 \%$ were born after 32 weeks of GA. Extremely low birth weight (birth weight $<1000$ grams; ELBW) babies accounted for $17.9 \%$ cases. Documented Apgar scores were available for only 91 cases. Among those, mean Apgar scores at one minute, five minutes and 10 minutes were 5.08 (SD 1.73), 6.45 (SD 1.41) and 7.56 (SD 1.19) respectively. Among those 91 cases, Apgar score at one minute was $<5$ in $31(22.1 \%)$ cases and Apgar score at 10 minutes was $<5$ in $13(14.3 \%)$ cases. Active resuscitation was required in 44 (31.4\%) cases at birth. Among those 44 cases $75.0 \%$ required bag and mask ventilation and remaining $25.0 \%$ required further steps of resuscitation. Other baseline characters of VLBW infants are presented in Table 1.

Mean maternal age was 24.66 (SD 4.79) years. Mean gravidity of mothers was 1.77 (SD 1.18). Possible maternal risk factors for birth of VLBW infants were evaluated. They are presented in Table 2. Occurrence of various morbidities among VLBW infants are presented in Table 3. Major morbidity was sepsis. Sepsis was diagnosed in 130 (92.9\%) cases. Only 22 cases (15.7\%) had blood culture proven sepsis. Non physiological hyperbilirubinemia was seen in $103(73.6 \%)$ cases. Among those 103 cases, 29 (28.2\%) cases required double volume exchange transfusion and remaining were managed with phototherapy alone. None of them developed kernicterus.

ANC: Antenatal check up; PROM: Premature rupture of membrane, PIH: Pregnancy induced hypertension. *Chronic medical diseases: Rheumatic heart disease 2, Chronic hypertension 2, Bronchial asthma 1, Systemic lupus erythematosus 1, Hyperthyroidism 1, severe anemia 1. \#Acute infections: Urinary tract infection 5, Pneumonia 1, Hepatitis E 1, mumps 1.

HMD: Hyaline membrane disease; UGI: Upper gastrointestinal; PDA: Patent ductus arteriosus; DIC: Disseminated intravascular coagulation; HIE: Hypoxic ischemic encephalopathy.

As far as the treatment is regarded; 139 (99.3\%) cases were treated with intravenous fluid. All of them were also given supplemental calcium in fluid. Those who developed hypocalcemia were given therapeutic 
doses of calcium. Oxygen supplementation was required in $131(93.6 \%)$ cases. All 130 (92.9\%) cases diagnosed to have clinical sepsis were treated with intravenous antibiotics and six $(4.3 \%)$ cases were also treated with antifungal agent. Among 68 (48.6\%) babies who had apnea spells, $60(88.2 \%)$ cases were treated with aminophylline. Cases with shock were treated with volume replacement and vasopressor support (dopamine and, or dobutamine). None of the $30(21.4 \%)$ cases who developed hyaline membrane disease (HMD) were treated with surfactant because of financial constraint. Assisted ventilation due to respiratory failure was required in $56(40.0 \%)$ cases. Mechanical ventilation was offered for $40(28.6 \%)$ cases. Remaining $16(11.4 \%)$ cases had to be managed with manual ventilation using resuscitation bag and endotracheal tube because of inadequate numbers of mechanical ventilator to meet the demand. Improved cases were transferred to intermediate care nursery and pediatric ward and later discharged home as per hospital protocol. There were $76(54.3 \%)$ cases that improved and discharged home. Death occurred in 49 (35.0\%) cases and $15(10.7 \%)$ cases left against medical advice. Among those 15 cases that left against medical advice, nine cases left despite improvement due to financial problems. Remaining six cases left due to poor chance of survival.

Primary causes of death were HMD in 25 cases, sepsis in 17 cases and hypoxic ischemic encephalopathy in four cases, accounting for $51.5 \%$, $34.7 \%$ and $8.1 \%$ of total deaths respectively. Other less common primary causes of death were necrotizing enterocolitis (NEC), intraventricular hemorrhage (IVH) and tracheoesophageal fistula in one $(0.7 \%)$ cases each. Mean duration of stay in NICU was 8.38 (SD 7.04) and median duration was seven days. Mean duration of total hospital stay was 14.37 (SD 12.20) days and median duration was 12 days. Median duration of hospital stay was 17.5 days for survivors and five days for expired cases.

Table 1: Showing Baseline Characters of Very Low Birth Weight Infants

\begin{tabular}{|l|c|c|}
\hline Characteristics & Number of Patients & Percentage (\%) \\
\hline Born in BPKIHS & 88 & 62.9 \\
\hline Born at home & 15 & 10.7 \\
\hline Twins & 34 & 24.3 \\
\hline Male sex & 85 & 60.7 \\
\hline Extremely low birth weight & 25 & 17.9 \\
\hline Appropriate for GA* & 105 & 75.0 \\
\hline Small for GA & 35 & 25.0 \\
\hline Cesarean delivery & 21 & 15.0 \\
\hline Requirement of active resuscitation at birth & 44 & 31.4 \\
\hline Cephalic presentation & 120 & 85.7 \\
\hline Exposed to antenatal steroid & 65 & 46.4 \\
\hline
\end{tabular}

*GA: Gestational age.

Table 2: Showing Risk Factors for Premature Delivery in Mothers of VLBW Infants.

\begin{tabular}{|l|c|c|}
\hline Risk factors & Number of Patients & Percentage (\%) \\
\hline Inadequate ANC visit & 134 & 95.7 \\
\hline Antepartum hemorrhage & 40 & 28.6 \\
\hline Twin pregnancy & 34 & 24.3 \\
\hline PROM & 33 & 23.6 \\
\hline Bad obstetric history & 18 & 12.9 \\
\hline PIH & 18 & 12.9 \\
\hline Maternal age $<20$ years & 12 & 8.6 \\
\hline Chronic medical diseases* & 9 & 6.4 \\
\hline Acute infections & 8 & 5.7 \\
\hline Grand multiparity & 6 & 4.3 \\
\hline Clinical chorioamnionitis & 6 & 4.3 \\
\hline Oligohydramnios & 4 & 2.9 \\
\hline Polyhydramnios & 3 & 2.1 \\
\hline Maternal age $>35$ years & 2 & 1.4 \\
\hline Infertility & 2 & 1.4 \\
\hline
\end{tabular}


Table 3: Showing Morbidities of VLBW Infants.

\begin{tabular}{|c|c|c|}
\hline Morbidity & Number of Patients & Percentage $(\%)$ \\
\hline Clinical sepsis & 108 & 77.1 \\
\hline Non Physiological Jaundice & 103 & 73.6 \\
\hline Apnoea & 68 & 48.6 \\
\hline Shock & 60 & 42.9 \\
\hline Hypoglycemia & 55 & 39.3 \\
\hline Anemia & 41 & 29.3 \\
\hline Hypocalcemia & 35 & 25.0 \\
\hline HMD & 30 & 21.4 \\
\hline UGI bleeding & 28 & 20.0 \\
\hline Culture positive sepsis & 22 & 15.7 \\
\hline PDA & 21 & 15.0 \\
\hline $\mathrm{DIC}$ & 21 & 15.0 \\
\hline Hypothermia & 17 & 12.1 \\
\hline Feed intolerance & 15 & 10.7 \\
\hline Necrotizing enterocolitis & 12 & 8.6 \\
\hline Acute renal failure & 12 & 8.6 \\
\hline HIE & 11 & 7.9 \\
\hline Retinopathy of prematurity & 8 & 5.7 \\
\hline Seizure & 7 & 5.0 \\
\hline Meningitis & 5 & 3.6 \\
\hline Bronchopulmonary dysplasia & 5 & 3.6 \\
\hline Intraventricular hemorrhage & 4 & 2.9 \\
\hline Pulmonary hemorrhage & 4 & 2.9 \\
\hline
\end{tabular}

\section{Discussion}

Preterm deliveries of VLBW babies are of major concern because of maximum perinatal morbidity and mortality found in this group ${ }^{3}$. The retrospective analysis of maternal antenatal profile in this study represents the various high risk factors responsible for preterm delivery of VLBW babies. Most common factor in this study was inadequate ANC visits. Lack of adequate antenatal care has been reported as a common factor leading to preterm birth by various studies $6,7,8$. So, encouraging pregnant women to have adequate ANC visits will reduce the burden of VLBW births. Antepartum hemorrhage (APH) was the second most common factor associated with preterm delivery in this study, accounting for $28.6 \%$ cases. There is a strong association between preterm labor and $\mathrm{APH}^{3,8}$. In a study by Kayastha et al. at another hospital of Nepal, APH was present in $5.8 \%$ mothers of low birth weight infants 9 . In another Indian study, APH was present in $9.7 \%$ mothers of VLBW infants. ${ }^{3}$ In this study, APH was present in higher proportion of cases as compared to those studies. Twin pregnancy was the third most common factor associated with VLBW births in this study accounting for $24.3 \%$ cases. In a study by Roy et al. at India, $11.9 \%$ mothers of VLBW babies had multiple pregnancy ${ }^{3}$. In another study at Japan by Kusuda et al., 30.0\% of total VLBW infants were because of multiple pregnancy ${ }^{10}$.
Hence, twin pregnancy is an important determinant for VLBW. Other common associations with VLBW births in this study were premature rupture of membrane, bad obstetric history, pregnancy induced hypertension, maternal acute infections, maternal chronic diseases and maternal age less than 20 years. These factors has been seen to be associated with low and very low

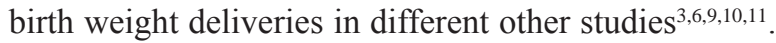
Maternal urinary tract infection, which was present in $3.6 \%$ mothers, was also a common association with VLBW births in this study. Roy et al. found urinary tract infection in $13.0 \%$ mothers of VLBW infants at India $^{3}$. Similarly; Kayastha et al. reported urinary tract infection to be present in $11.6 \%$ mothers of low birth weight infants at $\mathrm{Nepal}^{9}$.

So, in order to prevent the births of premature babies and decrease the burden of VLBW infants in our set up, few important measures should be taken. These measures are, increasing the coverage of ANC visits among pregnant women, early diagnosis and treatment of APH and pregnancy induced hypertension and discouraging the marriage and childbirth at too young age. Early diagnosis and treatment of acute infections and chronic medical diseases in mothers are also important measures to decrease the burden of VLBW 
births. When multiple pregnancies occur, preterm birth should be timely expected so as to manage appropriately. Mean birth weight of VLBW babies in this study was 1188.9 grams. In other similar studies at Japan, Saudi Arabia and Taiwan, the mean birth weights of VLBW babies were 1018 grams, 1133 grams and 1024 grams respectively ${ }^{10,11,12}$. Mean birth weight of VLBW babies is comparable to those from studies at different other parts of world ${ }^{10,11,12}$. The proportion of extremely low birth weight (ELBW, weighing less than 1000 grams) babies among VLBW babies in this study was $17.9 \%$. In a study by Roy et al at India, this proportion was $33.9 \% .^{3}$ In another Thai study, this proportion was $24.0 \%{ }^{13}$ In another study at Brazil, this proportion was $38.0 \%{ }^{14}$ Hence, we had lower proportion of ELBW babies among VLBW babies. One reason for this might be because of lower proportion of ELBW births in our part. But another more likely reason could be because less numbers of ELBW babies in our part are surviving enough to come to hospital. This needs to made clear by population based studies. The proportion of SGA babies in this study was $25.0 \%$. Similar to this study, the proportion was $25.8 \%$ in a study at Taiwan. ${ }^{12}$ The proportions of SGA babies among VLBW infants reported from studies done at Japan (36.0\%) and Brazil (39.0\%) are higher as compared to that from this study ${ }^{10,14}$.

In this study, $31.4 \%$ babies required active resuscitation at birth. This was similar to data from a study at Thailand ${ }^{3}$. In another study by Tsou et al. at Taiwan, $68.1 \%$ VLBW babies' required active resuscitation at birth. ${ }^{12}$ These figures implicate that a significant proportion of VLBW babies require active resuscitation at birth. So, preterm birth should always be conducted at centers where adequate facilities and equipments for neonatal resuscitation are available. Among 91 babies with available Apgar scores, mean Apgar scores at one, five and ten minutes were 5.08, 6.45 and 7.56 respectively in this study. This is similar to study by Mansouri et al. ${ }^{11}$ This shows that VLBW babies tend to have low Apgar scores. That is why large numbers of them require active resuscitation at birth.

Corticosteroids given to promote fetal lung maturation have become a mainstay in the management of women at risk of preterm birth. It has been found that the use of antenatal steroid to be associated with lower mortality rates in VLBW infants ${ }^{13}$. In this study, only $46.4 \%$ women received at least one dose of antenatal steroid. A large multicentric study at Japan also showed that only $41.0 \%$ mothers of VLBW infants received antenatal steroid ${ }^{10}$. Using antenatal steroid to treat women at risk of preterm birth might be an inexpensive method to improve survival of VLBW infants in our set up.
Sepsis was the most common morbidity encountered among VLBW babies in this study. Sepsis was diagnosed clinically in $77.1 \%$ cases but only $15.7 \%$ cases had blood culture proven sepsis. The proportion of cases with culture proven sepsis among VLBW infants in this study resembles the data from the similar studies done at developing countries like India and Kenya ${ }^{3,15}$. However, incidence of culture proven sepsis in this study is higher than the data from study done at developed country like Japan ${ }^{10}$. So, incidence of culture proven sepsis seems to be higher in our set up as compared to that in developed countries. Since any illness in VLBW infant may resemble sepsis clinically, we are probably over diagnosing sepsis clinically. This scenario is not much different in other parts of world also. For example, in the same Japanese study, $80.0 \%$ VLBW infants received antibiotics though there were only $8.0 \%$ cases of culture proven sepsis. ${ }^{10}$ The second most common morbidity in this study was non physiological hyperbilirubinemia which was diagnosed in $73.6 \%$ cases. This data resembles those from studies done at Saudi Arabia and India ${ }^{11,16}$. Since hyperbilirubinemia requiring intervention is such a common morbidity among VLBW infants, there is a need for evaluation of prophylactic therapies, which would prevent hyperbilirubinemia and would decrease the need as well as cost of intervention. The third most common problem was apnea, which occurred in almost half of the cases. In a study by Tsou et al. at Taiwan, $66.1 \%$ VLBW infants had apnoea ${ }^{12}$. Therefore apnea monitoring should be an integral component of care of VLBW infants.

HMD was diagnosed in $21.4 \%$ of VLBW infants in this study, which is similar to data from an Indian study. ${ }^{3}$ HMD was diagnosed in $54.0 \%$ to $79.3 \%$ VLBW infants in different other studies done at Japan, Saudi Arabia, Taiwan, and Malaysia ${ }^{10,11,12,17}$. Hence, HMD is a common problem among VLBW infants. However, it seems to be less common in South Asia region as compared to other parts of Asia, especially developed countries $^{3,10,11,12,17}$. In this study, 15.0\% VLBW infants were diagnosed to have patent ductus arteriosus (PDA). This figure is much less than the figures given by different other studies, where PDA was diagnosed in $24.3 \%$ to $36.0 \%$ VLBW babies ${ }^{10,18,19}$. This difference may be because we detected only clinically significant PDA by clinical examination alone. We did not perform echocardiography routinely in all VLBW infants. So, clinically insignificant PDA might have been missed in this study. Anemia was another common problem diagnosed in $29.3 \%$ cases. Simiyu et al. detected anemia in $17.0 \% \mathrm{VLBW}$ infants ${ }^{15}$. So, anemia is common problem among VLBW infants, mainly because of repeated blood sampling and anemia of prematurity. 
Other common morbidities in this study were shock (42.9\%), severe hypothermia (12.1\%), and disseminated intravascular coagulation $(15.0 \%)$. These three morbidities frequently co-exist together and one may lead to another. Hypothermia was diagnosed in as high as $45.0 \%$ VLBW infants in a study done at even a developed country like United States. ${ }^{20}$ Metabolic problems like hypoglycemia and hypocalcemia were also common in this study which were diagnosed in $39.3 \%$ and $25.0 \%$ cases respectively. Venkataraman et al. detected hypocalcemia in 53.3\% VLBW infants in a study $^{21}$. So, VLBW infants should be routinely monitored for these metabolic problems. Feed intolerance was another problem in $10.7 \%$ VLBW infants in this study. Boo et al. detected feed intolerance in $64.4 \%$ of VLBW infants and they recommended that to promote tolerance of enteral feeds, intermittent orogastric feed should be started as soon as possible during first 72 hours of life in VLBW infants ${ }^{22}$. NEC was diagnosed in $8.6 \%$ VLBW infants in this study, which was similar to finding of other studies. ${ }^{13,19}$ In one study done at Turkey, NEC was diagnosed in $26.3 \%$ VLBW infants. ${ }^{23}$ It has been shown that oral probiotics reduce the incidence and severity of NEC in VLBW infants ${ }^{24}$. This needs to be confirmed by more studies and if found to be effective, it can be used in VLBW infants in our set up also. The incidence of IVH in this study was only $2.9 \%$. This figure is much less than those reported by many other studies., $3,10,17,19$ We don't have bedside ultrasound facility to routinely screen VLBW infants for IVH. So, we diagnosed IVH by cranial ultrasound only after strong clinical suspicion. Hence, we might have under diagnosed IVH in VLBW infants. Similarly, routine check up of all VLBW infants for retinopathy of prematurity (ROP) is not strictly done in our hospital because most patients do not come for follow up. That is why we probably under diagnosed ROP, which was diagnosed in only $10.5 \%$ of survivors. This is much less than those reported by other studies $(19.0 \% \text { to } 31.0 \%)^{3,10,19,25}$. We diagnosed this condition in those babies who stayed long enough in hospital. All VLBW infants, especially those who are born before 32 weeks gestation, should be strictly followed up and routinely subjected for eye evaluation. We diagnosed bronchopulmonary dysplasia in only $3.6 \%$ of cases, which is also much less than those reported by other studies $(12.6 \% \text { to } 33.0 \%)^{10,12,13,19,23}$. This might be simply because less numbers of VLBW infants who develop HMD and require mechanical ventilation are surviving enough to develop bronchopulmonary dysplasia in our set up.

Overall survival of VLBW babies in this study was $54.3 \%$. This survival rate is less than the survival rates reported by other studies done at different parts of world, where survival rates were $78.3 \%$ to $90.3 \%$. $^{1,3,10,12,19,23}$ In this study, lower survival rate in comparison to that of developed countries was because of reasons like poor affordability of parents, limited resources, lack of adequate equipments and ventilators, and limited availability of advanced services such as surfactant therapy and subspeciality services in the study hospital. For example, none of the HMD cases in this study could afford for the surfactant therapy. When needed, 16 cases did not get mechanical ventilator and they had to be managed by manual ventilation. Hence, more numbers of more equipped NICUs are needed in our country to improve survival of VLBW babies. In this study, common causes of death were HMD and sepsis accounting for $51.0 \%$ and $34.7 \%$ of the total deaths respectively. Due to lack of resources and financial constraints, none of the babies received either prophylactic or therapeutic surfactant therapy. That was the reason for large number of deaths due to hyaline membrane disease. In study by Sritipsukho et al. at Thailand, HMD was the most common cause of death $(47.0 \%)$ followed by sepsis $(20.0 \%) .{ }^{13}$ This is similar to the finding of this study. In one of the studies at Taiwan, the most common cause of death was sepsis. ${ }^{12}$ In another Malaysian study, three most common causes of death were HMD (33.2\%), sepsis (29.6\%) and IVH $(17.9 \%)^{17}$. Hence, common causes of death match with those of this study. The median duration of hospital stay among survivors in this study was 17.5 days, with a range of one to 51 days. In a Thai study, it was 31.5 days $^{13}$. In a Japanese study, mean duration of stay was 97 days ${ }^{10}$. In another study at Taiwan, the mean duration of hospitalization was 67.2 days $^{19}$. This difference is because, in resource rich countries, they keep the babies in hospital till babies reach appropriate weight for discharge as per hospital guideline. Hospitals in developing countries can not follow weight guideline for discharge strictly because of limited resources to be shared by many babies and poor affordability of parents for prolonged hospital stay.

\section{Conclusion}

Preterm deliveries of VLBW babies are of major concern because of maximum perinatal morbidity and mortality found in this group. Inadequate ANC visits, APH, multiple pregnancy, pregnancy induced hypertension, young maternal age and medical diseases in mothers are the common associations with VLBW births in our set up. Increasing the coverage of ANC visits, early diagnosis and treatment of APH and pregnancy induced hypertension and discouraging the childbirth at too young age, early diagnosis and treatment of acute infections and chronic medical diseases in mothers are important measures to decrease the burden of VLBW births. Using antenatal steroid to treat women at risk of preterm birth might be an inexpensive method to improve survival of VLBW infants in our set up. Common morbidities in VLBW 
babies are sepsis, HMD, apnea, hyperbilirubinemia, PDA, shock, anemia, hypoglycemia, hypothermia and hypocalcemia. Common culprits for death of VLBW babies are HMD and sepsis. Due to lack of adequate resources we are over diagnosing sepsis and under diagnosing conditions like IVH, PDA and ROP. Overall survival of VLBW babies in our settings is less. More numbers of well equipped NICUs and services like surfactant therapy are needed to improve survival of VLBW infants in our set up.

\section{Acknowledgement: None}

\section{Funding: None}

\section{Conflict of Interest: None}

\section{References}

1. Darlow BA, Cust AE, Donoghue DA. Improved outcomes for very low birth weight infants: evidence from New Zealand national population based data. Arch Dis Child Fetal Neonatal Ed 2003; 88:F23-F28.

2. Gurubacharya SM, Gurubacharya RL. An overview of neonatal admissions at College of Medical Sciences (COMS). J Nepal Paediatr Soc 2007; 27(2):73-4.

3. Roy KK, Baruah J, Kumar S, Malhotra N, Deorari AK, Sharma JB. Maternal antenatal profile and immediate neonatal outcome in VLBW and ELBW Babies. Indian J Pediatr 2006; 73(8):669-73.

4. Carvalho MD, Gomes MASM. Mortality of very low birth weight preterm infants in Brazil: reality and challenges. J Pediatr (Rio J) 2005; 81(1 Suppl): S111-S118.

5. McIntrire DD, Bloom SL, Casey BM, Leveno KJ. Birth weight in relation to morbidity and mortality among newborn infants. N Engl J Med 1999; 340(16):1234-8.

6. Mavalankar DV, Gray RH, Trivedi CR. Risk factors for term low birth weight in Ahmedabad, India. Int J Epidemiol 1992 Apr; 21(2):263-72.

7. Vega J, Saez G, Smith M, Agurto M, Morris NM. Risk factors for low birth weight and intrauterine growth retardation in Santiago, Chile. Rev Med Chil 1993 Oct; 121(10):1210-9.

8. Ferraz EM, Gray RH, Cunha TM. Determinant of preterm delivery and intrauterine growth retardation in north-east Brazil. Intl J Epidemiol 1990 Mar; 19(1):101-8.

9. Kayastha S, Tuladhar H. Study of low birth weight babies in Nepal Medical College. Nepal Med Coll J 2007 Dec; 9(4):266-9.
10. Kusuda S, Fujimura M, Sakuma I, Aotani H, Kabe K, Itani Y, Ichiba H, Matsunami K, Nishida H. Morbidity and mortality of infants with very low birth weight in Japan: center variation. Pediatrics 2006 Oct; 118(4):1131-9.

11. Mansouri HA. Perinatal factors and neonatal outcome of very low birth weight and extremely premature babies at KAUH. Bahrain Med Bull 2001 Jun; 23(2):66-70.

12. Tsou KI, Tsao PN. The morbidity and survival of very-low-birth-weight infants in Taiwan. Acta Paediatr Taiwan 2003 Nov-Dec; 44(6):349-55.

13. Sritipsukho S, Suarod T, Sritipsukho P. Survival and outcome of very low birth weight infants born in a University Hospital with level II NICU. J Med Assoc Thai 2007; 90(7):1323-9.

14. DeMello FB, deAlmeida MFB, dosSantos AMN, Costa HPF, Miyoshi MH, Amaro ER. Factors associated with survival of very-low-birth-weight infants in a Brazilian fee-paying maternity in the 1990s. J Trop Pediatr 2007 Jun; 53(3):153-7.

15. Simiyu DE. Morbidity and mortality of low birth weight infants in the new born unit of Kenyatta National Hospital, Nairobi. East Afr Med J 2004 Jul; 81(7):367-74.

16. Narang A, Kumar P, Kumar R. Neonatal jaundice in very low birth weight babies. Indian J Pediatr 2001 Apr; 68(4):307-9.

17. Boo NY. Outcome of very low birth weight neonates in a developing country: experience from a large Malaysian maternity hospital. Singapore Med J 1992 Feb; 33(1):33-7.

18. K Herrman, C Bose, $\mathrm{K}$ Lewis, M Laughon. Spontaneous closure of the patent ductus arteriosus in very low birth weight infants following discharge from the neonatal unit. Arch Dis Child Fetal Neonatal Ed 2009; 94:F48-F50.

19. Chang SC, Lin CH, Lin YJ, Yeh TF. Mortality, morbidity, length and cost of hospitalization in very-low-birth-weight infants in the era of National Health Insurance in Taiwan: a medical center's experience. Acta Paediatr Taiwan 2000 Nov-Dec; 41(6):8-12.

20. Loughead MK, Loughead JL, Reinhart MJ. Incidence and physiologic characteristics of hypothermia in the very low birth weight infant. Pediatr Nurs1997 Jan-Feb; 23(1):11-5.

21. Venkataraman PS, Blick KE, Fry HD, Rao RK. Postnatal changes in calcium-regulating hormones in very low birth weight infants. Effect of early neonatal hypocalcemia and intravenous calcium infusion on serum parathyroid hormone and 
calcitonin homeostasis. Am J Dis Child1985 Sep; 139(9):913-6.

22. Boo NY, Soon CC, Lye MS. Risk factors associated with feed intolerance in very low birth weight infants following initiation of enteral feeds during the first 72 hours of life. J Trop Pediatr 2000 Oct; 46(5):272-7.

23. Atassay B, Giinlemez A, Unal S, Arsan S. Outcomes of very low birth weight infants in a newborn tertiary center in Turkey, $1997-2000$. Turk J Pediatr 2003 Oct-Dec; 45(4):283-9.
24. Lin HC, Su BH, Chen AC, Lin TW, Tsai CH, Yeh $\mathrm{TF}$, Oh W. Oral probiotics reduce the incidence and severity of necrotizing enterocolitis in very low birth weight infants. Pediatrics 2005 Jan; 115(1):1-4.

25. Nair PM, Ganesh A, Mitra S, Ganguly SS. Retinopathy of prematurity in VLBW and extreme LBW babies. Indian J Pediatr 2003 Apr; 70(4):3036. 